\section{'I've got hay-fever and my mouth is stinging!'}

\author{
S. Rose, ${ }^{1}$ R. Weld-Moore, ${ }^{2}$ N. Ghazali ${ }^{3}$ and L. Newman ${ }^{4}$
}

IN BRIEF
- Awareness of the condition of oral
allergy syndrome can facilitate diagnosis
through a positive skin prick testing in
the presence of a suggestive history.
- Management is by allergen avoidance.
In serious and refractory cases,
referral to a regional allergy clinic is
recommended.

\begin{abstract}
A case of oral allergy syndrome is presented. Crossreactivity of pollens with some fruit/vegetables causes immediate lgEmediated symptoms localised to the mouth. Diagnosis is suspected from positive skin prick testing in the presence of a suggestive history. Management is by allergen avoidance. In serious and refractory cases, referral to a regional allergy clinic is recommended.
\end{abstract}

\section{CASE REPORT}

A 42-year-old lady was referred to the maxillofacial department by her general dental practitioner with a nine month history of recurrent painful oral 'ulcerations', believing them to be caused by lichen planus. The onset was characterised by pain described as a 'stinging sensation' which occurred frequently on the left side of the oral cavity, which was the preferred side for chewing when eating. Sometimes this was accompanied by an 'itchy sensation' causing her to rub the areas causing 'blisters/ulcers', which persisted between one to three days in duration. The onset of symptoms occurred on immediate contact with raw fruits or vegetables, including apples, melon and raw potato. She denied any other symptoms relating to her skin.

Relevant medical history included hayfever and severe latex allergy for which she was prescribed an autoinjectable adrenalin (Epipen, $0.3 \mathrm{mg}$ ). She was also undergoing investigation for possible food allergy. There was a family history of atopy, as other family members are severely allergic to peanuts, developing anaphylaxis on contact with them.

'Senior House Officer, ${ }^{2}$ Trust Doctor, ${ }^{3 *}$ Specialist Registrar in Oral \&t Maxillofacial Surgery, ${ }^{4}$ Consultant Oral \&t Maxillofacial Surgeon, Maxillofacial Unit, Queen Victoria Hospital, Holtye Road, East Grinstead, RH19 3DZ

*Correspondence to: Miss Naseem Ghazali

Email: naseemghazali@doctors.org.uk

\section{Refereed Pape}

Accepted 24 August 2011

DOI: 10.1038/sj.bdj.2011.863

${ }^{\circledR}$ British Dental Journal 2011; 211: 369-370
Oral examination revealed a small healing ulcer on the left buccal mucosa (Fig. 1) and an isolated healing lesion with a fine white halo resembling desquamative mucosa on the labial mucosa (Fig. 2). These lesions had developed upon contact with raw apples the day before. There were no extensive dental restorations adjacent to the areas of the lesion.

The differential diagnoses considered were oral allergy, lichen planus, lichenoid reaction, major aphthous ulceration and pemphigoid/pemphigus. The patient was prescribed an oral benzydamine mouthwash $0.15 \%$ for symptomatic control while waiting for the results of the allergy testing. Investigations requested include routine blood investigations encompassing haematinics and an allergy test. Incisional biopsy was considered pending the allergy and blood test results.

Blood investigations were within normal range. The Fresh Fruit Skin Prick allergy test was carried out and the results revealed allergies to raw apples, raw potatoes, latex, cats, dust, peanuts, almonds, hazelnuts, grass and tree pollen, suggesting sensitisation to these items. With this finding and a highly suggestive history, oral allergy syndrome (OAS) was considered. Definitive management involved reinforcing advice on allergen avoidance and the need to use the Epipen in the event of anaphylaxis. The patient declined to have an incisional biopsy or further allergy testing, preferring to manage her symptoms simply through avoidance. Allergen

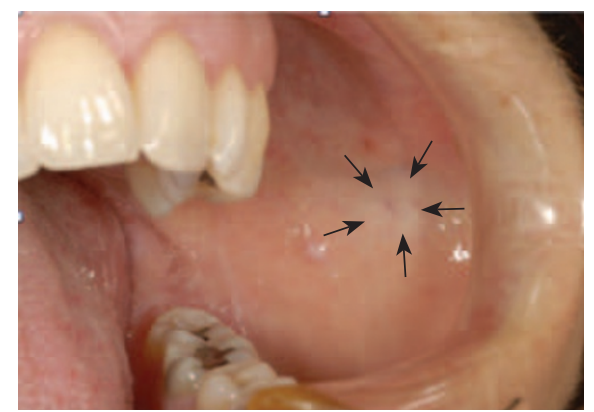

Fig. 1 Healing ulcer on the left buccal mucosa (marked out by blue arrows)

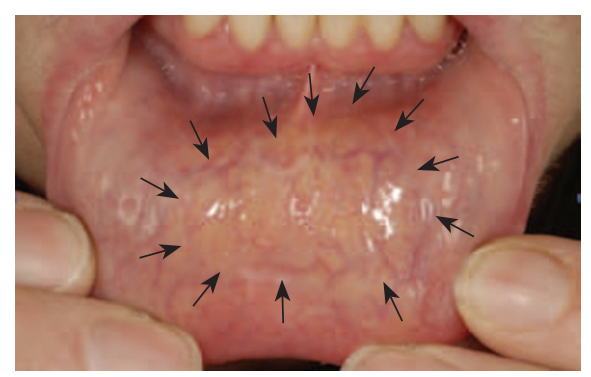

Fig. 2 White halo lesion (marked out with blue arrows) on the labial mucosa

avoidance successfully kept her symptoms at bay and subsequent clinical examinations found normal appearing oral mucosa throughout, including in those areas where the fine white halo had previously been.

\section{COMMENT}

The case presented highlights a condition that should be considered by the dental practitioner in patients with similarly presenting symptoms. OAS is an immunoglobulin-E (IgE)-mediated, immediate allergic reaction restricted most of the times in the oral mucosa. ${ }^{1-5}$ It is characterised by 
itching, swelling, and sometimes stinging pain of the oral tissues and lips as a result of eating certain raw vegetables, fruit and nuts. ${ }^{1-3,5}$ However, when the triggering plant foods are cooked, symptoms do not occur due to alteration of the antigen by the cooking process, although some antigens are resistant to heating. ${ }^{1,3,5}$ In our case, the persistence of oral ulcers may be secondary to frictional trauma to overcome the sensation of stinging and/or itchiness present.

Patients affected with OAS tend to suffer from pollinosis, a condition where symptoms of rhinoconjunctivitis and/or asthma occur as a result of inhalation of pollen, to which the individual is sensitised. ${ }^{3,5}$ The symptoms of OAS experienced in patients with pollinosis is the result of IgE crossreactivity to plant foods containing shared antigens to the pollen. ${ }^{1-5}$ Hence, OAS is increasingly referred to as the "pollenfood allergy syndrome. ${ }^{1-3,5}$ The common plant food reported to show crossreactivity with pollen is summarised in Table 1 . A subtype of OAS is the latex-fruit syndrome, which is characterised by allergy to fresh fruit or vegetables following sensitisation with the antigen related to latex powder inhalation. ${ }^{1}$

It is suggested that OAS may represent the most common food allergy in adults. ${ }^{2}$ However, the actual prevalence of OAS is unknown although it is suggested to be up to $10 \%$ of the population. ${ }^{4,5}$ The OAS is considered as a class 2 type of food allergy, where sensitisation is through respiratory exposure and not through conventional gastrointestinal tract sensitisation, as the food allergens of OAS are readily dissolved and digested by digestive enzymes. ${ }^{1-3}$ There are several types of pollinosis antigens causing OAS. The two most common pollinosis allergen groups causing OAS are the Bet $\mathrm{v} 1$ and Bet $v 2 .^{1-3}$ The Bet $v 1$ group of allergens, including birch, alder and white oak, show crossreactivity to many plant food including apples, cherry, hazelnut, celery and strawberry. The Bet v 2 group or 'profilin' allergens include mugwort, birch, sunflower, olive and latex show crossreactivity to many common fruits and vegetables including pineapple, sweet orange, banana, tomato, carrot, peach, apple and pear.
While OAS is recognised as a worldwide problem, geographical distribution of local flora producing pollen along with the geographical differences in dietary habit can influence the development and prevalence of different types of OAS. ${ }^{1,3,5}$ Birch pollensensitised patients in central and northern Europe often react to fruits from the Rosaceae family, such as peach, apricot, pear and apple. ${ }^{1,6}$ In America where ragweed pollen allergy is common, crossreaction occurs with melon and banana. ${ }^{1,6}$ In Japan, patients allergic to Japanese cedar pollen react with tomatoes. ${ }^{1,6}$

The degree of OAS symptoms experienced depends upon the lability of the antigen. ${ }^{1-3}$ Allergens that induce OAS are degraded in the oral cavity and stomach by digestive enzymes, resulting in local oral symptoms without any systemic involvement. However, certain pollinosis allergens are not heat-labile, ${ }^{5}$ for example, celery, which contains the allergen Api g $1 .^{1}$ This allergen is a major food-induced anaphylaxis and is a cause of systemic allergic reaction in Switzerland. ${ }^{1}$ It is suggested that OAS can progress to systemic symptoms in $8.7 \%$ and more worryingly, to anaphylactic shock in $1.7 \%$ of cases. $^{2}$

The diagnosis of OAS can be confused with other types of adverse reactions to food. ${ }^{3}$ As a guide, non-toxic food reactions that involve immune mechanisms are termed allergy while non-immunological mediated adverse reaction is termed intolerance. Central to the diagnosis of OAS is the role of IgE, which mediates this condition. Both the commercial skin prick test and the fresh fruit skin prick test (FFSPT) have been used to diagnose OAS, although there is evidence that the FFSPT has comparatively better sensitivity and specificity. ${ }^{2}$ Other diagnostic tests include food challenges $^{5}$ and more specialised immunological tests including specific IgE testing based on the underlying food, basophil activation test and sulphidoleukotriene release assay. ${ }^{2}$

The management of OAS is by allergen avoidance, education and emergency management with injectable adrenalin. ${ }^{1,2,7}$ Immunotherapy to desensitise patients to the causative allergens has been considered in the management of
Table 1 Common plant food reported to show cross-reactivity with pollen (adapted from Kondo and Urisu ${ }^{1}$ )

\begin{tabular}{|l|l}
\hline Pollen & Plant food \\
\hline \multirow{5}{*}{ Birch } & $\begin{array}{l}\text { Rosaceae (apple, pear, cherry, } \\
\text { peach, plum, apricot) } \\
\text { Apiaceae (celery, carrot) } \\
\text { Solanaceae (potato) } \\
\text { Actinidiaceae (kiwifruit) } \\
\text { Betulaceae (hazelnut) } \\
\text { Chilipepper, mung bean }\end{array}$ \\
\hline \multirow{5}{*}{ Mugwort } & $\begin{array}{l}\text { Apiaceae (celery, carrot) } \\
\text { Anacardiaceae (mango) } \\
\text { Rosaceae (peach) }\end{array}$ \\
\hline Grass & $\begin{array}{l}\text { Cucurbitaceae (melon, watermelon) } \\
\text { Solanaceae (potato, tomato) } \\
\text { Actinidiaceae (kiwifruit) } \\
\text { Peanut }\end{array}$ \\
\hline \multirow{2}{*}{ Ragweed } & $\begin{array}{l}\text { Cucurbitaceae (melon, watermelon, } \\
\text { cantaloupe, cucumber, } \\
\text { courgette, banana) }\end{array}$
\end{tabular}

OAS. ${ }^{1,2,4,5}$ The two types of immunotherapy approaches that have been investigated are the subcutaneous immunotherapy and sublingual immunotherapy. The long-term outcomes of both techniques are unpredictable.

In our case presentation, the striking history, positive FFSPT and the positive response obtained with avoidance were highly suggestive of the diagnosis of OAS. It may be argued that an incisional biopsy may provide conclusive evidence to rule out other oral lesions but this would not provide definitive diagnosis of OAS. Detailed allergy testing and immunotherapy could be justified when food avoidance has failed and/or symptoms are severe.

1. Kondo Y, Urisu A. Oral allergy syndrome. Allergol Int 2009; 58: 485-491.

2. Webber C M, England R W. Oral allergy syndrome: a clinical, diagnostic and therapeutic challenge. Ann Allergy Asthma Immunol 2010; 104: 101-108.

3. Bartra J, Sastre J, del Cuvillo A et al. From pollinosis to food allergy. J Investig Allergol Clin Immunol 2009; 19 Suppl 1:3-10.

4. Ma S, Sicherer S H, Nowak-Wegrzyn A. A survey on the management of pollen-food allergy syndrome in allergy practices. J Allergy Clin Immunol 2003; 112: 784-788.

5. Konstantinou G N, Grattan C E H. Food contact hypersensitivity syndrome: the mucosal contact urticaria paradigm. Clin Exp Dermatol 2008; 33: 383-389.

6. Maeda N, Inomata N, Morita A, Kirino M, Ikezawa Z Correlation of oral allergy syndrome due to plantderived foods with pollen sensitization in Japan. Ann Allergy Asthma Immunol 2010; 104: 205-210.

7. Lieberman P, Nicklas R A, Oppenheimer J et al. The diagnosis and management of anaphylaxis practice parameter: 2010 update. J Allergy Clin Immunol 2010; 126: 477-480 